\title{
UN PROBLÈME ASYMPTOTIQUE EN CONTRÔLE PONCTUEL
}

\author{
JACQUES-LOUIS LIONS
}

\section{Introduction}

Soit $\Omega$ un ouvert borné de $\boldsymbol{R}^{n}$, de frontière $\Gamma$ régulière. Dans $\Omega$, on considère un système gouverné par une équation hyperbolique avec contrôle s'exercant en $b$ donné, $b \in \Omega$. Plus précisément on suppose que l'état du système est donné par la solution de

$$
\begin{gathered}
\varepsilon \frac{\partial^{2} y_{\varepsilon}}{\partial t^{2}}+\frac{\partial y_{\varepsilon}}{\partial t}-\Delta y_{\varepsilon}=v(t) \delta(x-b) \\
\text { dans } Q=\Omega \times] 0, T[,
\end{gathered}
$$

où $\varepsilon$ est donné $>0$ et est destiné à tendre vers zéro; $\delta(x-b)$ désigne la messe de Dirac au point $b$; le contrôle est $v=v(t) \in L^{2}(0, T)$. On ajoute à (1) les conditions aux limites et initiales

$$
\begin{aligned}
\left.y_{\varepsilon}=0 \quad \text { sur } \quad \Sigma=\Gamma \times\right] 0, T[ \\
y_{\varepsilon}(x, 0)=0, \quad \frac{\partial y_{\varepsilon}}{\partial t}(x, 0)=0 \quad \text { dans } \quad \Omega .
\end{aligned}
$$

Les équations (1), (2) et (3) définissent de manière unique l'état

$$
y_{\varepsilon}=y_{\varepsilon}(x, t, v)=y_{\varepsilon}(v)
$$

dont on sait (cf. J. L. Lions [6] ${ }^{1)}$ ) que

$$
y_{\varepsilon}(v) \text { est continue de }[0, T] \rightarrow L^{2}(\Omega) .
$$

On définit alors la fonction coût

$$
J_{\varepsilon}(v)=\int_{\Omega}\left[y_{\varepsilon}(x, T, v)-z_{d}(x)\right]^{2} d x+N \int_{0}^{T} v(t)^{2} d t,
$$

où

$$
z_{d} \text { est donné dans } L^{2}(\Omega), N \text { est donné positif. }
$$

${ }^{1}$ La première démonstration de ce fait, par des méthodes puissantes d'Analyse Harmonique, est dûe à Y. Meyer [8] et a été suivie de démonstrations «directes» par L. Nirenberg [9] et l'A. [6]. 
On introduit

$$
\mathscr{U}_{a d}=\text { ensemble convexe fermé non vide de } L^{2}(0, T)
$$

(l'ensemble des contrôles admissibles) et l'on considère le problème de contrôle optimal

$$
\inf J_{\varepsilon}(v), \quad v \in \mathscr{U}_{a d} .
$$

Ce problème admet une solution unique $u_{\varepsilon}$. Nous nous intéressons ici au comportement de $u_{\varepsilon}$ lorsque $\varepsilon \rightarrow 0$.

La situation formelle est «claire ». Le problème limite est un système parabolique dont l'état

$$
y=y(x, t, v)=y(v)
$$

est donné par

$$
\frac{\partial y}{\partial t}-\Delta y=v(t) \delta(x-b)
$$

avec

$$
\begin{aligned}
y=0 & \text { sur } \quad \Sigma, \\
y(x, 0)=0 & \text { dans } \Omega .
\end{aligned}
$$

La fonction coût est alors donnée par

$$
J(v)=\int_{\Omega}\left[y(x, T, v)-z_{d}(x)\right]^{2} d x+N \int_{0}^{T} v^{2} d t
$$

et on pourrait penser que le «problème limite» est

$$
\inf J(v), \quad v \in \mathscr{U}_{a d} .
$$

Mais il y a une difficulté et le « résultat» précédent n'est pas tout à fait correct. En effet il existe des $v_{0} \in L^{2}(0, T)$ tels que $y\left(T, v_{0}\right) \notin L^{2}(\Omega)$.

Si donc on prend pour $\mathscr{U}_{a d}$ l'ensemble $\left\{v_{0}\right\}, v_{0}$ comme ci-dessus, le problème limite (15) n'a pas de sens. En fait on doit introduire l'espace

$$
\mathscr{U}^{(n)}(0, T)=\left\{v \mid v \in L^{2}(0, T), \quad y(T, v) \in L^{2}(\Omega)\right\} ;
$$

cet espace, muni de la norme de graphe

$$
\left(\int_{0}^{T} v^{2} d t+\int_{\Omega} y(x, T, v)^{2} d x\right)^{1 / 2}
$$

est un espace de Hilbert étudié dans J. L. Lions [4]-[5], Li Ta-tsien [1]-[2], Shi Shu-Chung [10], J. Simon [11]. Cet espace (à une équivalence de norme près) est indépendant de $b$, de $\Omega$, et même ( $\mathrm{Li}$ Ta-tsien, loc. cit.) de l'opérateur $\Delta$ que peut être remplacé par un opérateur elliptique du $2^{\text {ème }}$ ordre à coefficients réguliers sans que l'espace $\mathscr{U}^{(n)}$ change. Formellement (il y a des difficultés techniques, étudiées dans J. Simon [11]) l'espace $\mathscr{U}^{(n)}(0, T)$ coïncide avec l'espace des $v \in L^{2}(0, T)$ telles que

$$
\int_{0}^{T} \int_{0}^{T}(2 T-(t+s))^{-n / 2} v(t) v(s) d t d s<\infty .
$$


Le passage à la limite indiqué formellement ci-dessus devient alors correct si l'on suppose que

$$
\mathscr{U}_{a d} \cap \mathscr{U}^{(n)}(0, T) \neq \emptyset
$$

le problème limite étant

$$
\inf J(v), \quad v \in \mathscr{U}_{a d} \cap \mathscr{U}^{(n)}(0, T) .
$$

Nous donnons au № 2 ci après des indications, assez rapides, sur la démonstration de ce résultat après avoir donné au № 1 un résultat de perturbation singulière pour un système hyperbolique-parabolique.

\section{Perturbation singulière pour un système hyperbolique-parabolique}

Avec les notations de l'Introduction, on considère l'équation

$$
\begin{gathered}
\left.\varepsilon \frac{\partial^{2} \Phi}{\partial t^{2}}+\frac{\partial \Phi}{\partial t}-\Delta \Phi=0 \quad \text { dans } \quad Q=\Omega \times\right] 0, T[ \\
\Phi=0 \quad \text { sur } \quad \Sigma=\Gamma \times] 0, T[ \\
\mid \begin{array}{ll}
\Phi(x, 0)=0 & \text { dans } \quad \Omega, \\
\varepsilon \frac{\partial \Phi}{\partial t}(x, 0)=g(x) & \text { dans } \Omega
\end{array}
\end{gathered}
$$

avec $g$ donnée dans $L^{2}(\Omega)$.

Pour chaque $\varepsilon>0$, (1.1), (1.2) et (1.3) admet une solution unique $\Phi_{\varepsilon}$, telle que

$$
\Phi_{\varepsilon} \quad \text { est continue de }[0, T] \rightarrow H_{0}^{1}(\Omega),{ }^{1)}
$$

$$
\frac{\partial \Phi_{\varepsilon}}{\partial t} \quad \text { est continue de }[0, T] \rightarrow L^{2}(\Omega) \text {. }
$$

On va montrer que, lorsque $\varepsilon \rightarrow 0$,

$$
\Phi_{\varepsilon} \rightarrow \Phi \text { dans } L^{2}\left(0, T ; H_{0}^{1}(\Omega)\right) \text { faible, }
$$

où $\Phi$ est la solution de

$$
\begin{gathered}
\frac{\partial \Phi}{\partial t}-\Delta \Phi=0 \text { dans } Q \\
\Phi=0 \text { sur } \Sigma, \\
\Phi(x, 0)=g(x) \text { dans } \Omega .
\end{gathered}
$$

Remarque 1.1. Il y a donc un phénomène un peu inhabituel pour $t=0$, les conditions (1.3) devenant (1.9).

${ }^{1}$ On utilise les notations habituelles des espaces de Sobolev, comme par exemple dans J. L. Lions et E. Magenes [7]. 
La situation est entièrement différente si, au lieu de (1.3), on considère,

$$
\Phi_{\varepsilon}(x, 0)=g^{0}(x), \quad \frac{\partial \Phi_{\varepsilon}}{\partial t}(x, 0)=g^{1}(x)
$$

Cette fois la convergence de $\Phi_{\varepsilon}$ a lieu au sens (1.6), vers $\Phi$ solution de (1.7), (1.8) et, au lieu de (1.9) :

$$
\Phi(x, 0)=g^{0}(x) .
$$

Pour cette situation, cf. M. Zlámal [12], J. L. Lions [3], chapitre 6, paragraphe 5.

Remarque 1.2. Puisque $\Phi_{\varepsilon}(0) \neq \Phi(0)$ (si $g \neq 0$ !) il y a une couche limite pour $t=0$. Si l'on suppose que

et si l'on pose

$$
g \in H^{3}(\Omega), \quad g, \Delta g=0 \quad \text { sur } \quad \Gamma
$$

alors

$$
w_{\varepsilon}=\Phi_{\varepsilon}-\Phi+g e^{-t / \varepsilon}
$$

$$
\left\|w_{\varepsilon}\right\|_{L^{\infty}\left(0, T ; H_{0}^{1}(\Omega)\right)}+\left\|w_{\varepsilon}^{\prime}\right\|_{L^{2}\left(0, T ; L^{2}(\Omega)\right)} \leqq C \sqrt{\varepsilon}
$$

où $C$ ne dépend que de la norme de $g$ dans $H^{3}(\Omega)$.

Démonstration de (1.6). On introduit :

$$
w_{\varepsilon}(t)=\int_{0}^{T} \Phi_{\varepsilon}(\sigma) d \sigma
$$

et on intègre (1.1) de 0 à $t$; il vient (on écrit $\Phi^{\prime}, \Phi^{\prime \prime}$ au lieu de $\partial \Phi / \partial t, \partial^{2} \Phi / \partial t^{2}$ ) :

$$
\varepsilon \Phi_{\varepsilon}^{\prime}(t)-g+\Phi_{\varepsilon}(t)-\Delta w_{\varepsilon}(t)=0 .
$$

On prend le produit scalaire de (1.13) par $-\Delta \Phi_{\varepsilon}(t)$.

On pose :

$$
\begin{gathered}
a(\Phi, \gamma)=\int_{\Omega} \nabla \Phi \nabla \gamma d x, \quad a(\Phi, \Phi)=a(\Phi), \\
(\Phi, \gamma)=\int_{\Omega} \Phi \gamma d x, \quad(\Phi, \Phi)=|\Phi|^{2} .
\end{gathered}
$$

Alors

ou encore

$$
\varepsilon a\left(\Phi_{\varepsilon}^{\prime}(t), \Phi_{\varepsilon}(t)\right)+a\left(\Phi_{\varepsilon}(t)\right)+\left(\Delta w_{\varepsilon}(t), \Delta \Phi_{\varepsilon}(t)\right)=\left(g,-\Delta \Phi_{\varepsilon}(t)\right),
$$

$$
\frac{1}{2} \frac{d}{d t}\left[\varepsilon a\left(\Phi_{\varepsilon}(t)\right)+\left|\Delta w_{\varepsilon}(t)\right|^{2}\right]+a\left(\Phi_{\varepsilon}(t)\right)=\left(g,-\Delta \Phi_{\varepsilon}(t)\right) .
$$

Intégrant (1.14) de 0 à $t$, il vient :

$$
\varepsilon a\left(\Phi_{\varepsilon}(t)\right)+\left|\Delta w_{\varepsilon}(t)\right|^{2}+2 \int_{0}^{T} a\left(\Phi_{\varepsilon}(\sigma)\right) d \sigma=2\left(g,-\Delta w_{\varepsilon}(t)\right) .
$$


Il résulte de (1.15) que, lorsque $\varepsilon \rightarrow 0$,

$$
\mid \begin{aligned}
& \Phi_{\varepsilon}\left(\text { respectif } \sqrt{\varepsilon} \Phi_{\varepsilon}\right) \quad \text { demeure dans un ensemble borné de } \\
& L^{2}\left(0, T ; H_{0}^{1}(\Omega)\right) \quad\left(\text { respectif de } L^{\infty}\left(0, T ; L^{2}(\Omega)\right)\right),
\end{aligned}
$$

$$
\mid \begin{aligned}
& \Delta w_{\varepsilon} \text { demeure dans un borné de } L^{\infty}\left(0, T ; L^{2}(\Omega)\right), \text { et donc } \\
& w_{\varepsilon} \text { dans un borné de } L^{\infty}\left(0, T ; H^{2}(\Omega) \cap H_{0}^{1}(\Omega)\right) .
\end{aligned}
$$

On peut alors extraire une sous suite, encore notée $\Phi_{\varepsilon}, w_{\varepsilon}$, telle que

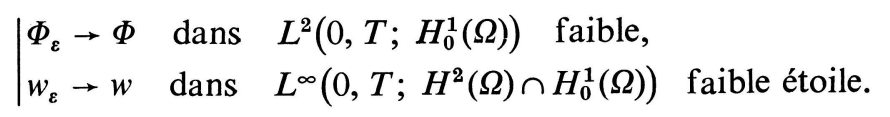

D’après (1.12) on a

$$
w(t)=\int_{0}^{t} \Phi(\sigma) d \sigma
$$

de sorte que (1.13) donne

d'où le résultat désiré.

$$
\Phi(t)-\Delta \int_{0}^{t} \Phi(\sigma) d \sigma=g
$$

\section{Problème de contrôle ponctuel perturbé}

Nous reprenons maintenant le problème (9) de l'Introduction, qui admet une solution unique $u_{\varepsilon}$, caractérisée par le système d'optimalité suivant :

$$
\begin{gathered}
\mid \begin{array}{l}
\varepsilon y_{\varepsilon}^{\prime \prime}+y_{\varepsilon}^{\prime}-\Delta y_{\varepsilon}=u_{\varepsilon} \delta(x-b), \\
\varepsilon p_{\varepsilon}^{\prime \prime}-p_{\varepsilon}^{\prime}-\Delta p_{\varepsilon}=0 \quad \text { dans } Q,
\end{array} \\
\mid \begin{array}{l}
y_{\varepsilon}(x, 0)=y_{\varepsilon}^{\prime}(x, 0)=0, \\
p_{\varepsilon}(x, T)=0, \quad \varepsilon p_{\varepsilon}^{\prime}(x, T)=-\left(g_{\varepsilon}(x, T)-z_{d}(x)\right) \quad \text { dans } \quad \Omega, \\
y_{\varepsilon}=p_{\varepsilon}=0 \quad \text { sur } \Sigma,
\end{array}
\end{gathered}
$$

et

$$
\mid \begin{aligned}
& \int_{0}^{T}\left(p_{\varepsilon}(b, t)+N u_{\varepsilon}(t)\right)\left(v(t)-u_{\varepsilon}(t)\right) d t \geqq 0 \quad \forall v \in \mathscr{U}_{a d}, \\
& u_{\varepsilon} \in \mathscr{U}_{a d}
\end{aligned}
$$

Remarque 2.1. On a ${ }^{1)}$

$$
p_{\varepsilon}(b, t) \in L^{2}(0, T)
$$

de sorte que (2.4) a un sens.

\footnotetext{
${ }^{1}$ C'est la propriété «duale» de (5) Introduction, démontrée directement par L. Nirenberg [9].
} 
Sous l'hypothèse (19) de l'Introduction, le problème (20) de l'Introduction admet une solution unique $u$, qui est caractérisée par le système d'optimalité suivant:

et

$$
\begin{gathered}
\mid \begin{array}{l}
y^{\prime}-\Delta y=u \delta(x, b), \\
-p^{\prime}-\Delta p=0 \quad \text { dans } \quad Q,
\end{array} \\
\mid \begin{array}{l}
y(x, 0)=0, \\
p(x, T)=y(x, T)-z_{d}(x) \text { dans } \quad \Omega, \\
y=p=0 \text { sur } \Sigma,
\end{array}
\end{gathered}
$$

$$
\mid \begin{aligned}
& \int_{0}^{T}(p(b, t)+N u(t))(v(t)-u(t)) d t \geqq 0 \quad \forall v \in \mathscr{U}_{a d} \cap \mathscr{U}^{(n)}(0, T), \\
& u \in \mathscr{U}_{a d} \cap \mathscr{U}^{(n)}(0, T) .
\end{aligned}
$$

Remarque 2.2. On a montré dans J. L. Lions [4]—-[5] que

$$
p(b, t) \in\left(\mathscr{U}^{(n)}(0, T)\right)^{\prime}
$$

de sorte que (2.9) a un sens.

Remarque 2.3. Dans un sens que l'on va préciser, le système (2.6)...(2.9) est limite du système (2.1)...(2.4). La condition

$$
p(x, T)=y(x, T)-z_{d}(x) \text { sur } \Omega
$$

provient des conditions (2.2) pour $p_{\varepsilon}$, comme l'explique le №1 (avec, par rapport au №1, un changement de signe dû au fait que pour $p_{\varepsilon}$ on intègre en $t$ dans le sens rétrograde).

On va montrer que, lorsque $\varepsilon \rightarrow 0$,

$$
\mid \begin{aligned}
& u_{\varepsilon} \rightarrow u \text { dans } L^{2}(0, T) \text { faible, } \\
& y_{\varepsilon}(., T) \rightarrow y(., T) \text { dans } L^{2}(\Omega) \text { faible. }
\end{aligned}
$$

On commence par observer que (grâce au fait que $\mathscr{U}_{a d} \cap \mathscr{U}^{(n)}(0, T)$ n'est pas vide) $J_{\varepsilon}\left(u_{\varepsilon}\right) \leqq C$ lorsque $\varepsilon \rightarrow 0$. Donc

$$
\mid \begin{array}{lll}
u_{\varepsilon} & (\text { respectif } & \left.y_{\varepsilon}(., T)\right) \\
(\text { respectif de } & \left.L^{2}(\Omega)\right) .
\end{array}
$$

On peut donc extraire une sous suite, encore notée $u_{\varepsilon}, y_{\varepsilon}(., T)$, telle que

$$
\mid \begin{aligned}
& u_{\varepsilon} \rightarrow u^{*} \text { dans } L^{2}(0, T) \text { faible, } \\
& y_{\varepsilon}(., T) \rightarrow \eta \text { dans } L^{2}(\Omega) \text { faible. }
\end{aligned}
$$

Il résulte alors du №1 que

$$
p_{\varepsilon} \rightarrow p^{*} \quad \text { dans } \quad L^{\infty}\left(0, T ; H_{0}^{1}(\Omega)\right) \text { taible }
$$


où $p^{*}$ est caractérisé par

$$
\mid \begin{aligned}
& -\frac{\partial p^{*}}{\partial t}-\Delta p^{*}=0 \quad \text { dans } \quad Q, \\
& p^{*}(x, T)=\eta(x)-z_{d}(x) \text { dans } \Omega, \\
& p^{*}=0 \quad \text { sur } \quad \Sigma .
\end{aligned}
$$

On peut vérifier que

$$
p_{\varepsilon}(b, t) \rightarrow p^{*}(b, t) \quad \text { dans } \quad\left(\mathscr{U}^{(n)}(0, T)\right)^{\prime} \text { faible. }
$$

Considérons (2.4) pour $v \in \mathscr{U}_{a d} \cap \mathscr{U}^{(n)}(0, T)$, que nous écrivons

$$
\int_{0}^{T} p_{\varepsilon}(b, t) v(t) d t+N \int_{0}^{T} u_{\varepsilon}(t) v(t) d t \geqq \int_{0}^{T} p_{\varepsilon}(b, t) u_{\varepsilon}(t) d t+N \int_{0}^{T} u_{\varepsilon}(t)^{2} d t
$$

Mais par application de la formule de Green,

$$
\mid \int_{0}^{T} p_{\varepsilon}(b, t) u_{\varepsilon}(t) d t=\int_{Q}\left(\varepsilon y_{\varepsilon}^{\prime \prime}+y_{\varepsilon}^{\prime}-\Delta y_{\varepsilon}\right) p_{\varepsilon} d x d t=\left(y_{\varepsilon}(T), y_{\varepsilon}(T)-z_{d}\right)
$$

de sorte que (2.17) s'écrit

$$
\int_{0}^{T}\left(p_{\varepsilon}(b, t)+N u_{\varepsilon}(t)\right) v(t) d t \geqq\left(y_{\varepsilon}(T), y_{\varepsilon}(T)-z_{d}\right)+N \int_{0}^{T} u_{\varepsilon}^{2} d t .
$$

On peut passer à la limite dans (2.19). Il vient :

$$
\mid \begin{aligned}
& \int_{0}^{T}\left(p^{*}(b, t)+N u^{*}(t)\right) v(t) d t \geqq\left(\eta, \eta-z_{d}\right)+N \int_{0}^{T}\left(u^{*}\right)^{2} d t \\
& \forall v \in \mathscr{U}_{a d} \cap \mathscr{U}^{(n)}(0, T) .
\end{aligned}
$$

Notons maintenant que

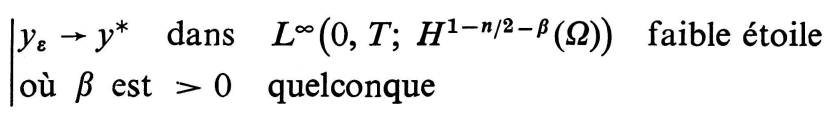

où $y^{*}$ est la solution de

$$
\mid \begin{aligned}
& \frac{\partial y^{*}}{\partial t}-\Delta y^{*}=u^{*} \delta(x, b) \\
& y^{*}(x, 0)=0 \quad \text { dans } \quad \Omega, \quad y^{*}=0 \quad \text { sur } \quad \Sigma .
\end{aligned}
$$

Admettant un instant ce point, il en résulte que

$$
\eta=y^{*}(x, T) \text { dans } \Omega
$$

de sorte que (2.22), (2.15) et (2.20) coïncide avec le système d'optimalité (2.6)...(2.9), lequel admet une solution unique. Donc $u^{*}=u, y^{*}=y, p^{*}=p$ et l'on a, en particulier, le résultat $(2.11)$, sous réserve de vérifier (2.21) et (2.22). 
Tous revient à montrer que

$$
\mid \begin{array}{ll}
y_{\varepsilon} & \text { demeure dans un borné de } L^{\infty}\left(0, T ; H^{1-n / 2-\beta}(\Omega)\right) \\
\text { où } & \beta \text { est }>0 .
\end{array}
$$

Posons : $A=-\Delta$, avec les conditions aux limites de Dirichlet.

Nous utilisons les puissances fractionnaires $A^{s}, s \in \boldsymbol{R}$.

Prenons le produit scalaire de $(2.1)_{1}$ par $A^{-\vartheta} y_{\varepsilon}^{\prime}, \vartheta$ à choisir.

Il vient :

$$
\frac{\varepsilon}{2} \frac{d}{d t}\left|A^{-\vartheta / 2} y_{\varepsilon}^{\prime}\right|^{2}+\left|A^{-\vartheta / 2} y_{\varepsilon}^{\prime}\right|^{2}+\frac{1}{2} \frac{d}{d t}\left|A^{(1-\vartheta) / 2} y_{\varepsilon}\right|^{2}=\left(A^{-\vartheta / 2} \delta(x, b), A^{-\vartheta / 2} y_{\varepsilon}^{\prime}\right) u_{\varepsilon}(t) .
$$

Choisissons $\vartheta$ de manière que

$$
A^{-\vartheta / 2} \delta(x-b) \in L^{2}(\Omega) \text {. }
$$

Comme $\delta(x-b) \in H^{-\sigma}(\Omega)$ si $\sigma>n / 2$, on a

$$
A^{-\vartheta / 2} \delta(x-b) \in H^{\vartheta-\sigma}(\Omega) \quad \text { d'où }(2.26) \quad \text { si }=\sigma>n / 2 .
$$

Avec ce choix de $\vartheta$, le $2^{\text {ème }}$ membre de (2.25) est majoré par

d'où résulte que

$$
C\left|u_{\varepsilon}(t)\right|\left|A^{-\vartheta / 2} y_{\varepsilon}^{\prime}\right|
$$

$A^{-9 / 2} y_{\varepsilon}^{\prime}$ demeure dans un borné de $L^{2}\left(0, T ; L^{2}(\Omega)\right)$

et que

$$
A^{(1-9) / 2} y_{\varepsilon} \text { demeure dans un borné de } L^{2}\left(0, T ; L^{2}(\Omega)\right)
$$

d'où résulte (2.24).

\section{Bibliographie}

[1] Li TA-TSIEn [Li DA-QIAN]: Propriétés d'espaces fonctionnels intervenant en contrôle optimal. C. R. Acad. Sci. Paris Sér. A 289, 1979, 687-690.

[2] Li TA-Tsien [Li DA-QIAN]: Propriétés d'espaces fonctionnels et problèmes de contrôle optimal de systèmes gouvernés par les équations paraboliques. - C. R. Acad. Sci. Paris Sér. A 290, 1980, 697-700.

[3] Lions, J. L.: Perturbations singulières dans les problèmes aux limites et en contrôle optimal. - Lecture Notes in Mathematics 323. Springer-Verlag, Berlin-Heidelberg-New York, 1973.

[4] Lions, J. L.: Function spaces and optimal control of distributed systems. - [Lecture notes.] Universidade Federal do Rio de Janeiro, Istituto de Matematico, Rio de Janeiro, 1980.

[5] Lions, J. L.: Some methods in the mathematical analysis of systems and their control. - Science Press, Beijing/Gordon and Breach, Science Publishers, Inc., New York, 1981.

[6] Lions, J. L.: Some questions in the optimal control of distributed systems. - Actes du congrès international tenu à l'occasion du 50ème anniversaire de l'Institut Mathématique Steklov, Moscou, Sept. 24-26, et Léningrad, Sept. 27-28, 1984. (A paraître.) 
[7] Lions, J. L., et E. Magenes: Problèmes aux limites non homogènes et applications. 1. - Travaux et recherches mathématiques 17. Dunod, Paris, 1968.

[8] MeYer, Y.: Étude d'un modèle mathématique issu du contrôle des structures spatiales déformables. - Nonlinear partial differential equations and their applications. Collège de France Seminar. VII. Editeurs: H. Brézis et J. L. Lions. Research Notes in Mathematics 122. Pitman Advanced Publishing Program, Boston-LondonMelbourne, 1985, 234-242.

[9] Nirenberg, L.: Communication personnelle.

[10] Sнiн Shu-Chung: Sur une classe d'espaces fonctionnels intervenant en contrôle optimal. C. R. Acad. Sci. Paris Sér. A 290, 1980, 761-764.

[11] Simon, J.: Contrôle de la solution d'une équation parabolique avec donné ponctuelle. J. Systems Sci. Math. Sci. 3:1, 1983, 1-27.

[12] Zlámal, M.: Sur un problème mixte pour des équations hyperboliques avec un petit paramètre - Czechoslovak Math. J. 10, 1960, 83-122 (en Russe).

Collège de France

F-75231 Paris Cedex 05

France

Reçu le 17 septembre 1984 